\title{
Musical pitch identification by absolute pitch possessors
}

\author{
KEN'ICHI MIYAZAKI \\ Niigata University, Niigata, Japan
}

\begin{abstract}
Musical pitch identification was investigated in two experiments in which absolute pitch (AP) possessors and nonpossessors categorized tones presented in isolation into predetermined pitch classes. Stimuli consisted of 60 different tones per octave (at intervals of 20 cents). The experiments were designed to minimize the possibility that subjects could use strategies other than AP in performing the task. The results clearly differentiated AP possessors from nonpossessors in accuracy and speed of responding. Those subjects who had AP could categorize the tones quite consistently by using musical qualities of the tones (tone chroma). However, they did not respond uniformly to all stimuli; they responded more accurately and quickly to some musically important tones in a C-major mode (C, E, or G). On the other hand, those who had no AP showed almost random response patterns. In the absence of a tonal context, they could not use tone chroma, but only tone height. It is argued that tone chroma should be defined as the musical characteristics of tones in a tonal context, and that AP possessors are unique in that they can perceive it absolutely in the absence of any musical context. Although AP was believed to be very rare, it was proved here that a phenomenally large proportion of the subjects tested had AP. The correlation was observed between AP possession and early musical training that started at the age of 3 to 5 .
\end{abstract}

Absolute pitch (AP) is referred to as the faculty for identifying or producing musical pitch without recourse to any reference tones. Given a reference tone, people who have no AP but some musical experience can tell the note name of a target tone more or less accurately on the basis of relative pitch (musical interval sense). However, with no reference tones available, they are unsuccessful in identifying isolated tones and can differentiate only a small number of pitch categories (Pollack, 1952). This limit of resolution in single-interval paradigms, often regarded as an example of the so-called "magical number seven phenomenon" (Miller, 1956), can be accounted for in the context of memory models (e.g., Durlach \& Braida, 1969; Siegel, 1974). In contrast, some people who possess AP can accomplish the task successfully without any reference tones.

The topic of AP has interested psychologists for a long time (e.g., Révész, 1913; Stumpf, 1883; Wellek, 1963). Some investigators have argued that AP possessors are rare even among musicians and that $\mathrm{AP}$ is, therefore, a special endowment. The problem of whether AP is innate or learned has been a major source of controversy (e.g., Bachem, 1937; Neu, 1947), and many investigations have been carried out in an attempt to form AP through training (Brady, 1970; Cuddy, 1968, 1970; Meyer, 1899; Wedell, 1934). Although the problem of the genesis of AP is still at issue (Ward \& Burns, 1982), the present study was not concerned with this problem;

Address correspondence to Ken'ichi Miyazaki, Department of Psychology, College of General Education, Niigata University, 8050, Igarashi-2-no-cho, Niigata 950-21, Japan. instead the emphasis was on the perceptual and cognitive aspects of AP and its behavioral outcome. Curiously, there seem to be relatively few investigations that have dealt with AP from a psychophysical and/or cognitive psychological standpoint.

In early experiments of AP, experimenters often presented stimulus tones in isolation by using musical instruments, most often the piano, and the subjects who were assumed to have AP were instructed to tell note names (Bachem, 1937; Petran, 1932; Wellek, 1938). In such a procedure, the stimuli are inevitably restricted to tones of the traditional musical tone system (i.e., 12 tones in an octave). Therefore, it is possible that musically experienced subjects who have no AP but have an excellent musical interval sense (relative pitch) can be persuaded to rely on the strategy of using relative pitch, resulting in response patterns similar to those of true AP possessors. Furthermore, piano tones are considered to be inadequate stimuli for the AP experiment, because they are so familiar to musicians that any cues other than pitch can be put to use (Ward \& Burns, 1982). If the non-AP subjects who have extensive musical experience adopt such sophisticated strategies, their performance may be, at least qualitatively, indistinguishable from that of true AP possessors. Bachem (1937) pointed out that pseudo AP should be distinguished from true AP. To examine AP in the true sense of the word, researchers must exclude the possibility that the non-AP subjects will use those strategies based on relative pitch and timbral cues.

Two experiments on absolute musical pitch identification were carried out, in which subjects with AP or without AP were instructed to categorize tones presented 
in isolation into predetermined pitch classes. In terms of the remarks mentioned above, electronically generated complex tones were used as stimuli, and one semitone interval was divided into five logarithmically equal steps, resulting in a stimulus set of 60 different pitches per octave. In this way, it was anticipated that response distributions of pitch identification of true AP possessors could be clearly differentiated from those of non-AP subjects.

The purposes of this study were as follows: (1) to develop an experimental task that clearly separates AP possessors from nonpossessors, (2) to evaluate how accurately AP possessors can categorize stimulus tones when a semitone is finely subdivided, and (3) to estimate the range and position of musical pitch categories of AP possessors along a frequency continuum.

\section{EXPERIMENT 1}

\section{Method}

Procedure. Stimulus tones were randomly presented to the subjects. The stimuli consisted of 60 different tones: one equally tempered semitone was divided into five logarithmically equal steps (at intervals of 20 cents). Each tone was displaced to one of three different octaves from trial to trial, and a tone on two consecutive trials differed by more than one octave. This procedure was to prevent the subjects from relying upon the strategy of using relative pitch in their judgments. In each trial, the subjects attempted to answer musical note names of the presented tones by neglecting their octave positions and by pressing corresponding keys of a computer console that was likened to a musical keyboard. Black marks were attached to the tops of some keys of the console to mimic the musical keyboard. When the presented tone sounded off-tune, the subjects were instructed to press a space bar of the console. After a response occurred, $6 \mathrm{sec}$ elapsed as an intertrial interval, and then another tone of the next trial was delivered. No feedback was given.

Five experimental sessions were carried out for each listener. In each session, 60 different tones were randomly presented once. One session took about 10 to $12 \mathrm{~min}$, and rest periods of sufficient length were inserted between sessions.

Stimuli. Stimuli were sawtooth waves whose fundamental frequencies ranged from $130.8 \mathrm{~Hz}$ (C3) to $1046.5 \mathrm{~Hz}$ (C6), with the standard pitch of $440 \mathrm{~Hz}$ as A4. The envelope of the stimulus amplitude had a shape of fast attack and slow decay. The attack time was about $5 \mathrm{msec}$, and the total duration of the stimulus was about $1.5 \mathrm{sec}$.

Apparatus. The stimuli were generated, filtered, and shaped by a voltage-controlled oscillator (VCO), voltage-controlled filter (VCF), and voltage-controlled amplifier (VCA) module of a music synthesizer (Roland, SYSTEM-100M). Fundamental frequencies of the stimuli, cutoff frequencies of the VCF module, and gate timing were determined by a sequencer (Roland, CMU-800), which was controlled by a computer (NEC, PC-8801). This computer was also used for registering responses and calculating results. The output of the synthesizer was amplified by an amplifier (Victor, X-1000A) and presented to subjects through a loudspeaker (Yamaha, NS$1000 \mathrm{M}$ ) located in a quiet room. The overall level of tones was 70 to $75 \mathrm{~dB}(\mathrm{~A})$.

Subjects. Ten musically experienced listeners participated in the experiment as subjects. Most of them were students of the Department of Music Eduation of Niigata University and had taken piano lessons starting at 3 to 5 years of age. Whether they were AP possessors or not was not evident before the experiment, although a few of them indicated with reservations that they might have AP.

\section{Results}

The results showed that 7 of the 10 subjects possessed AP. All 7 could identify the tones fairly well, although the variability of the responses was somewhat different among the subjects. For the 7 subjects with AP, the proportion of responses with less than one semitone error was $94.1 \%$, ranging from $90.2 \%$ to $99.4 \%$; for the other 3 subjects, the mean was $27.2 \%$ and the range was $42.7 \%$ to $13.9 \%$. Thus, the former 7 subjects could be designated as AP possessors, and the latter 3 as non-AP subjects. All the AP subjects and 1 of the non-AP subjects had received musical training from an early age, whereas 2 of the non-AP subjects had no such training.

Figure 1 shows the overall scatter pattern of the responses obtained from the subjects designated as AP possessors; stimulus categories within an octave are represented on an abscissa (disregarding octave position), and response categories are represented on an ordinate. The size of each circle in the plot is proportional to the number of responses that occurred when the corresponding stimuli were presented. It is shown that most responses to each stimulus category occurred in the nearest corresponding response category. As a result, almost all responses are located on a diagonal line from the lower left to the upper right, indicating that the subjects could consistently classify the tones into corresponding musical pitch categories. A row of circles at the upper end of the plot indicates off-tune responses, which are mainly at the intermediate locations between correctly tuned musical pitches.

Figure 2 shows the frequency distribution patterns of responses of $1 \mathrm{AP}$ subject who did the task most consistently; in the figure, the number of responses of each category is plotted as a function of the frequency of the stimuli expressed in cents relative to $\mathrm{C} 3, \mathrm{C} 4$, or $\mathrm{C} 5$. One can see that the response distribution patterns of different musical pitch labels show extremely clear-cut categorization. It has already been pointed out that AP possessors perceive pitch categorically and that their performances are comparable to those of relative pitch subjects (Siegel \& Siegel, 1977).

For comparison, the results obtained from 2 non-AP subjects are shown in Figures 3a and 3b. The responses of Subject M.T. (Figure 3a) are widely dispersed, indicating that he could not identify note names at all. However, it appears that the responses of Subject K.M. (Figure 3b) are not evenly dispersed, but are roughly clustered from the lower left to the upper right. He, indeed, had no AP, but used all available means in executing the task successfully. He used, for example, the strain in the vocal organ and the relative pitch sense on the basis of some anchoring pitches, such as the extremes of his vocal range or particular pitches he could easily produce. As a result of his effort, he could respond as if he had AP, but very inaccurately. It must be mentioned, however, that in spite of his effort, his responses were so widely scattered that they could not compare with those of genuine AP possessors. 


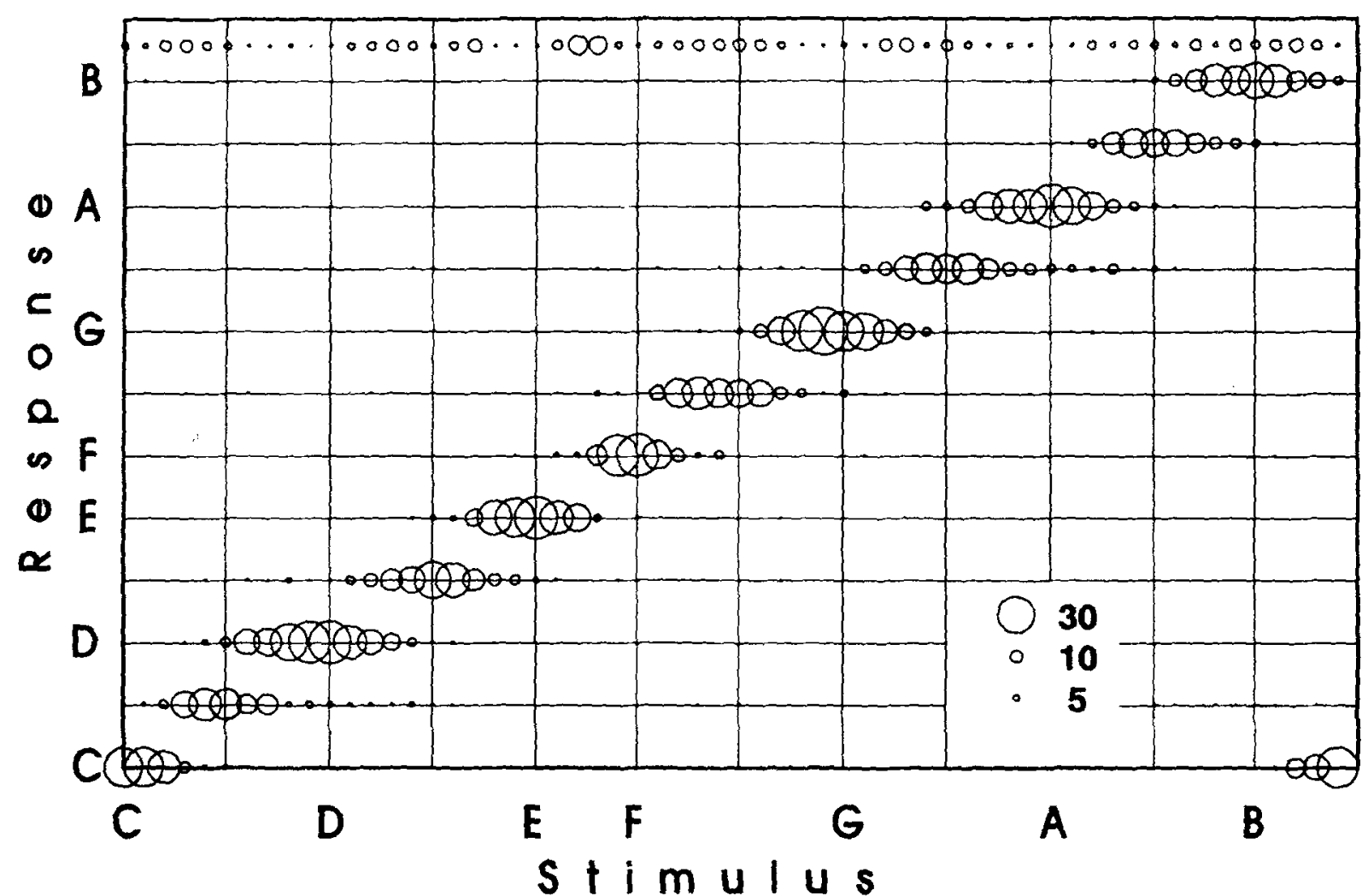

Figure 1. Scatter pattern of responses as a fonction of stimulus tones, viewed in the one-octave range for 7 subjects with absolute pitch (Experiment 1). The responses and the stimuli are expressed in musical note numes. The size of each circle is proportional to the aumber of times each response was made. The row of circles at the top represents off-tune responses.

It can be concluded from the results of Experiment 1 that the AP subjects tested in the experiment could consistently classify tones presented in isolation, which included many an intermediate pitch, into the traditional musical pitch categories. Some non-AP subjects could cope with the task by using some available cues, but these subjects were nevertheless far behind genuine AP subjects in accuracy. These results show that the task can clearly distinguish genuine AP subjects from non-AP subjects or pseudo-AP subjects.

\section{EXPERIMENT 2}

In the previous experiment, stimulus tones presented were at intervals of 20 cents, but response categories were restricted to a pitch set of the ordinary chromatic scale (at intervals of 100 cents). Some AP subjects claimed that they had difficulty deciding into which tonal categories they should classify an intermediate pitch between chromatic steps. These subjects felt that additional response categories were needed. Therefore, Experiment 2 was

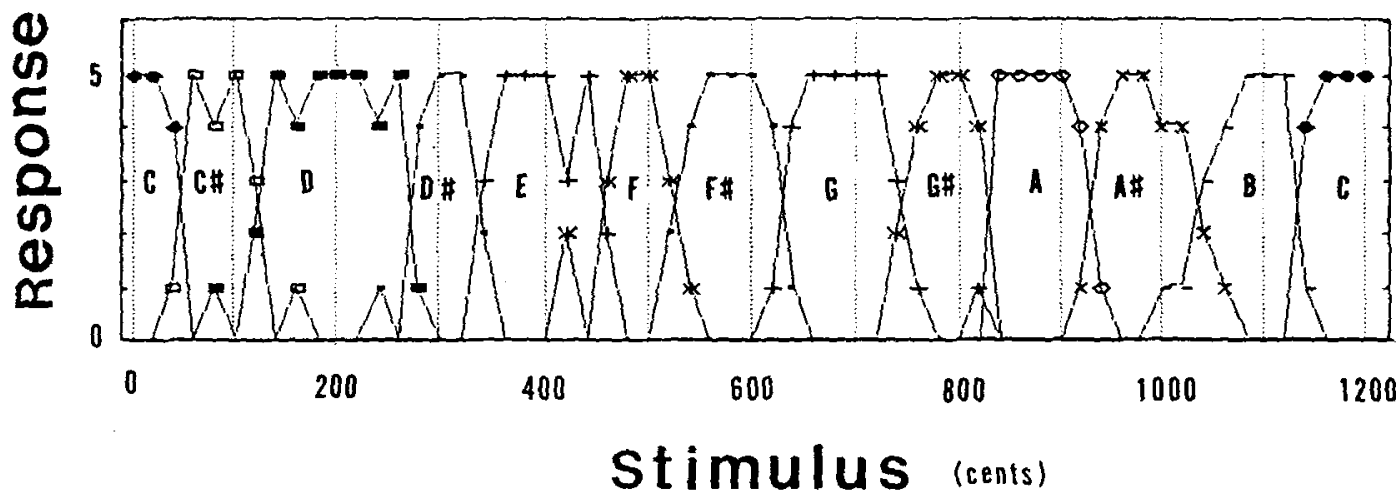

Figure 2. Number of times each response was made as a function of stimulus frequencies in the one-actave range for 1 possessor of absolute pitch (Experiment 1). The stimulus frequencies are expressed in cents relative to $C$ notes. 
(a)

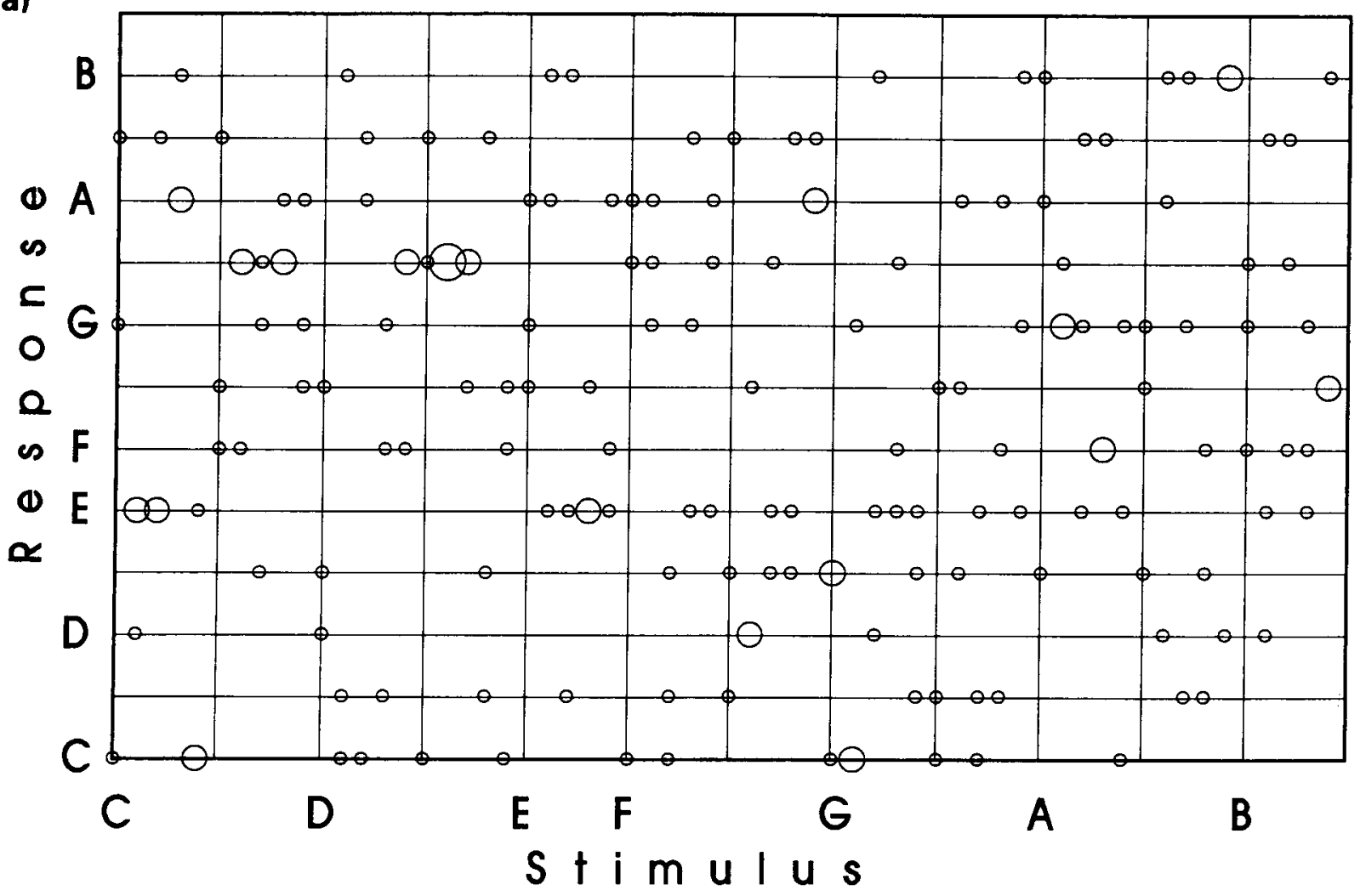

(b)

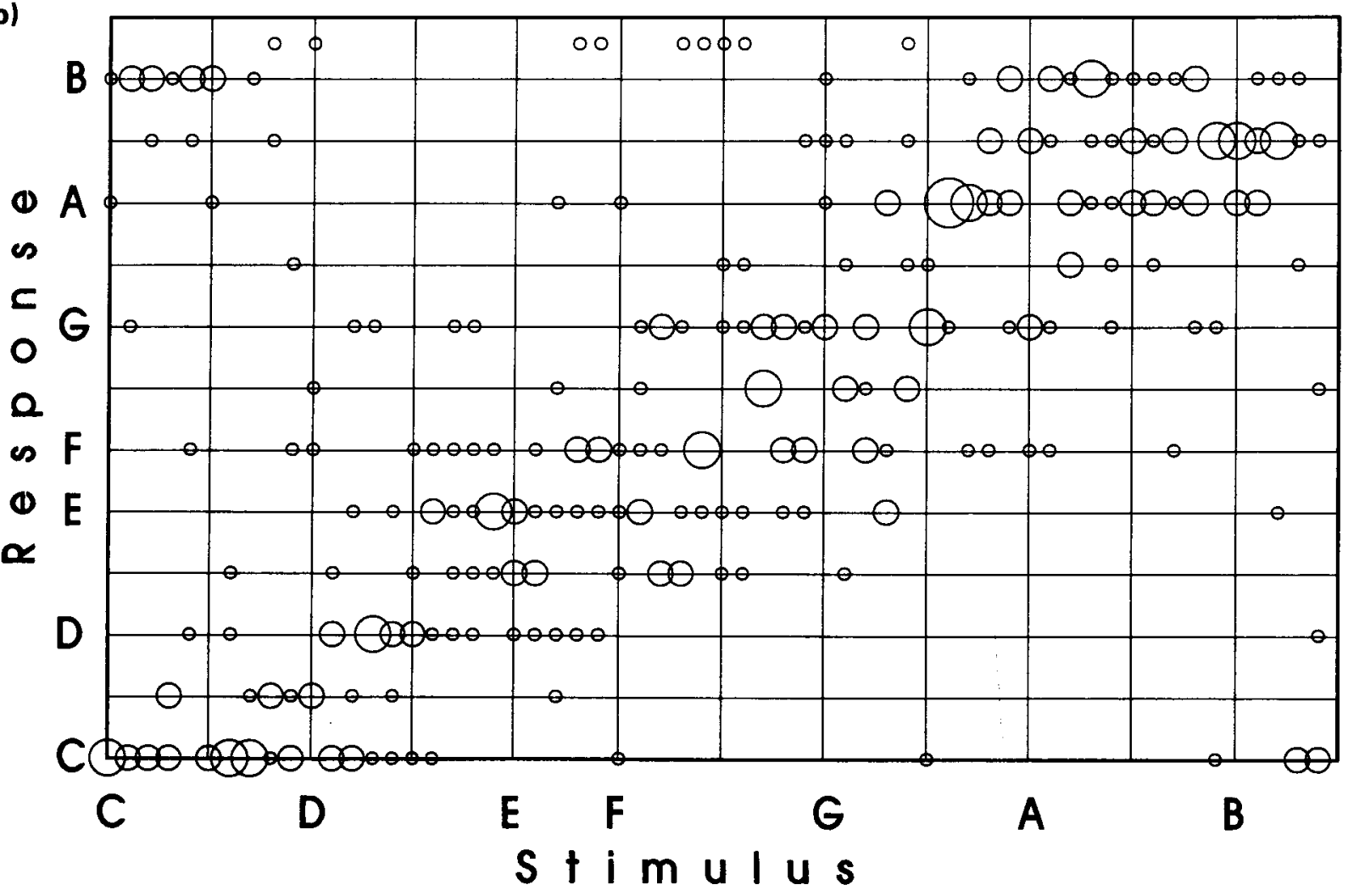

Figure 3. Scatter patterns of responses as a function of stimulus tones, viewed in the one-octave range for 2 individual subjects without absolute pitch (Experiment 1). The size of each circle is proportional to the number of times each response was made. 
performed to ascertain whether AP possessors could classify a microtonal pitch consistently into more finely divided perceptual categories.

\section{Method}

Procedure. Stimuli consisted of 180 different tones that were separated at logarithmically equal intervals in a range of three octaves; as in Experiment 1, one equally tempered semitone was divided into five logarithmically equal steps (at intervals of 20 cents). The tones of the consecutive trials differed by more than one octave to keep the subjects from relying on relative judgment from previously presented tones. In each trial, the subjects attempted to identify the presented tone by using a musical keyboard. If the pitch of the tone sounded acceptable as a musical note, they pressed a key corresponding to that note. If the subjects perceived the tone lower or higher than a musical note, they were to press first a corresponding key and then a lower or higher adjacent key (either a white or black key). Thus, three subcategories were differentiated for each ordinary musical note. The subjects were instructed to make responses as accurately and rapidly as possible, but not to sacrifice accuracy of response in favor of rapidity. The response time (RT), a time interval between the onset of a stimulus tone and the occurrence of a keypress, was measured. After the response occurred, 6 sec elapsed as an intertrial interval, and then another tone for the next trial was delivered. No feedback was given.

Each listener carried out two experimental sessions, in each of which 180 different kinds of pitch appeared once in pseudorandom order with constraints as described above. Short rest periods were inserted on occasion within the session. One session took about 35 to $40 \mathrm{~min}$.

Stimuli and Apparatus. The stimuli were piano-like tones generated by a module of a digital synthesizer (Yamaha, TX-816) based on a frequency-modulation technique for sound synthesis originated by Chowning (1973). Fundamental frequencies ranged from $130.8 \mathrm{~Hz}$ (C3) to $1034.5 \mathrm{~Hz}$ (20 cents lower than C6) with the standard pitch of $440 \mathrm{~Hz}$ as A4. The time envelope of the tone had a shape of fast attack and slow decay; the attack time was approximately $5 \mathrm{msec}$, and the total duration of the stimulus was about $1.5 \mathrm{sec}$. To respond, the subjects used a keyboard of another music synthesizer (Yamaha, DX-7) which generated no sounds. Stimulus presentation and registration of responses were carried out by a computer (NEC, PC-9801) through a Musical-Information-DigitalInterface (MIDI) bus and a MIDI processing unit (Roland, MPU401). The output of the synthesizer was amplified by an amplifier (Victor, X-1000A) and presented through a loudspeaker (Yamaha, NS-1000M) to subjects in a quiet room. The overall level of tones was about 70 to $75 \mathrm{~dB}(\mathrm{~A})$.

Subjects. Thirty-nine university students participated in the experiment. Their experience of musical practice was of various degrees. Fourteen of them were university students in a music education course and had begun piano lessons when 3 to 5 years old. The others had taken no formal music lessions, but had participated in various musical activities, such as chorus, orchestra, or pop band.

\section{Results}

Response distribution. On the basis of response accuracy, the subjects were divided into three subgroups: the precise $A P$ group (12 subjects), the imprecise $A P$ group (10 subjects), and the non- $A P$ group (17 subjects). The mean proportion of responses within a range of onesemitone error was $94.1 \%$ (ranging from $99.7 \%$ to $86.7 \%)$ for precise AP subjects, $69.1 \%(83.1 \%$ to $45.0 \%)$ for imprecise AP subjects, and $27.5 \%$ (34.4\% to $20.6 \%$ ) for non-AP subjects. A correlation was found between AP possession and early musical training; all subjects of the precise AP group had begun piano lessons at 3 to 5 years of age (4.1 years on average), whereas the number of subjects who had similar early musical training was 8 out of 10 imprecise AP subjects and only 5 out of 17 non-AP subjects.

The scatter pattern of all responses of the 12 precise AP possessors is shown in Figure 4a, where the responses are plotted relative to a stimulus continuum. The size of each circle in the figure represents the number of responses in each category. It can be seen from the figure that most responses are clustered along a diagonal line from the lower left to the upper right. One can also see that several responses are located in lines parallel to the diagonal. These are the so-called octave errors, which are correct for note names but incorrect for octave positions. The occurrence of the octave error is characteristic of AP possessors (Révész, 1913; Wellek, 1938).

The same data are replotted on a one-octave stimulusresponse plot in which only note names are considered (Figure $4 \mathrm{~b}$ ). It can be seen that the majority of the responses are "in tune," located on horizontal lines in Figure 4b. Compared with them, the number of "out-oftune" responses (lower or higher) is lower and cannot be regarded as clear-cut response categories as "in tune" responses are so long as the overall data of the AP group are considered.

One can find several extremely accurate individual AP possessors. The results of one such subject are shown in a collapsed one-octave range plot (Figure 5). The figure indicates that the "lower" and "higher" responses occurred consistently; for example, responses of "lower G" occurred mainly when the stimulus was in fact slightly lower than G. It may be suggested that good AP possessors can categorize more finely than can a standard tuning system (Ward, 1963).

The frequency distributions of the "in-tune" responses of the same subject are shown in Figure 6, from which one can see that "in-tune" responses occur in a very narrow range around an objectively correct pitch. It does not seem difficult for good AP possessors to reject a slightly mistuned pitch. Such extremely fine sensitivity of AP possessors to the tuning detail may be comparable to the good musical interval sense of experienced musicians (see Burns \& Ward, 1978).

The scatter pattern of all responses of the 17 non-AP subjects is shown in a three-octave range plot (Figure 7). In contrast to the responses of the AP group, these responses are widely scattered from the lower left to the upper right and are almost evenly dispersed in each octave range. These results clearly show that non-AP subjects could not consistently tell the musical note name of the presented tone; instead, they made judgments solely on the imprecise basis of the pitch height or timbre-like qualities of tones. This is considered one phenomenon of limited absolute judgments due to the absence of mnemonic anchors.

Response time. The time delay from the stimulus onset to the occurrence of response is expected to provide 


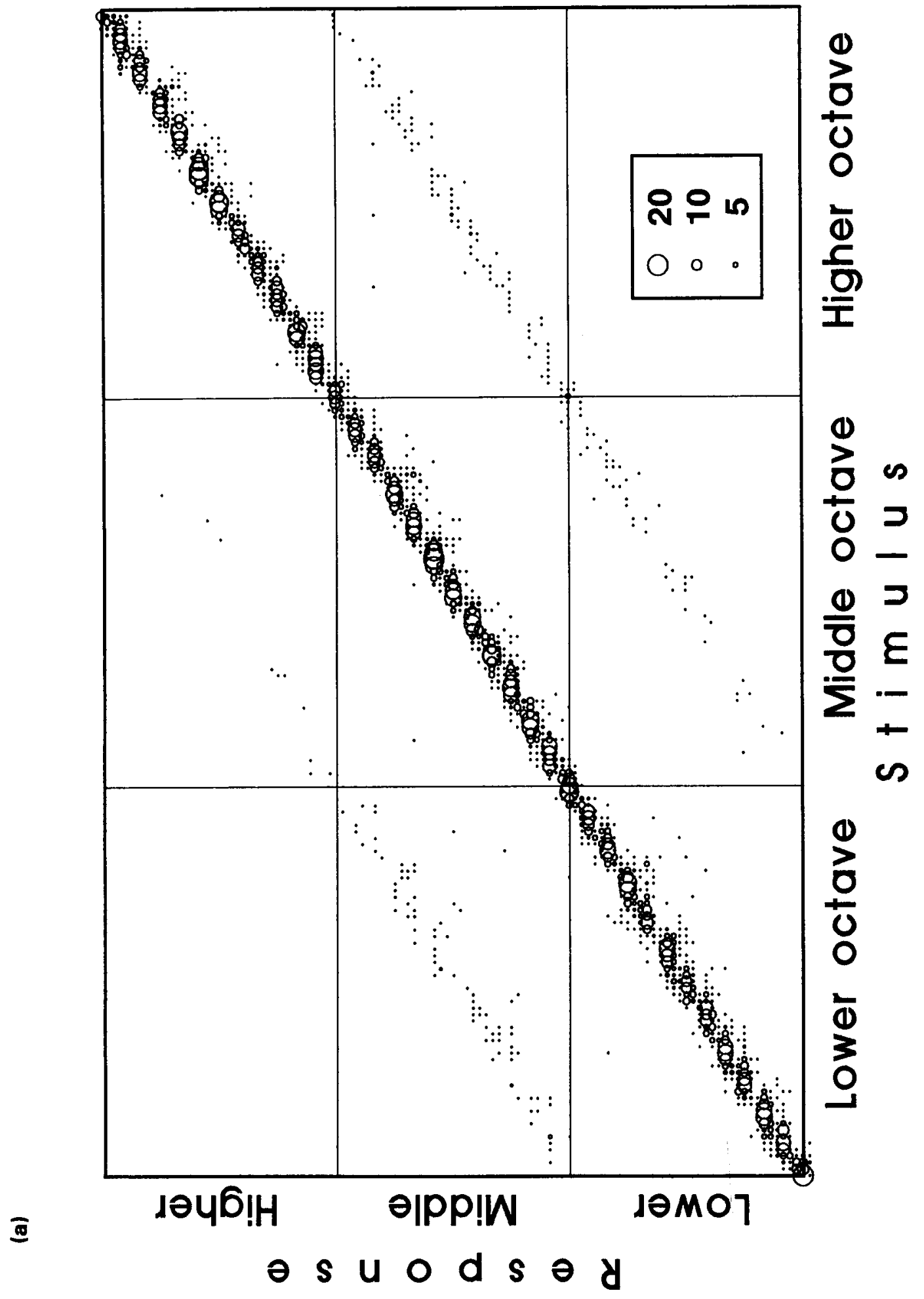




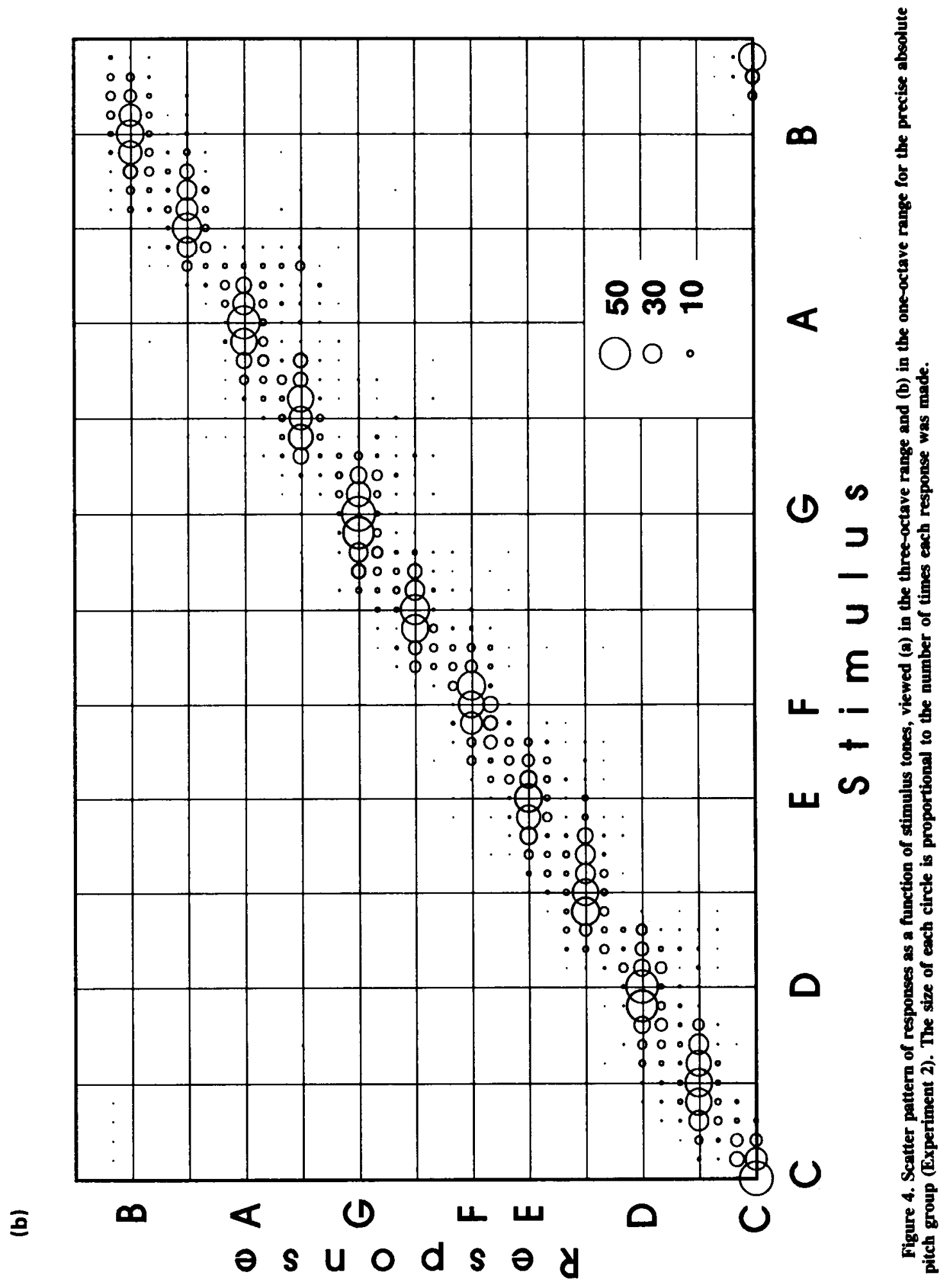




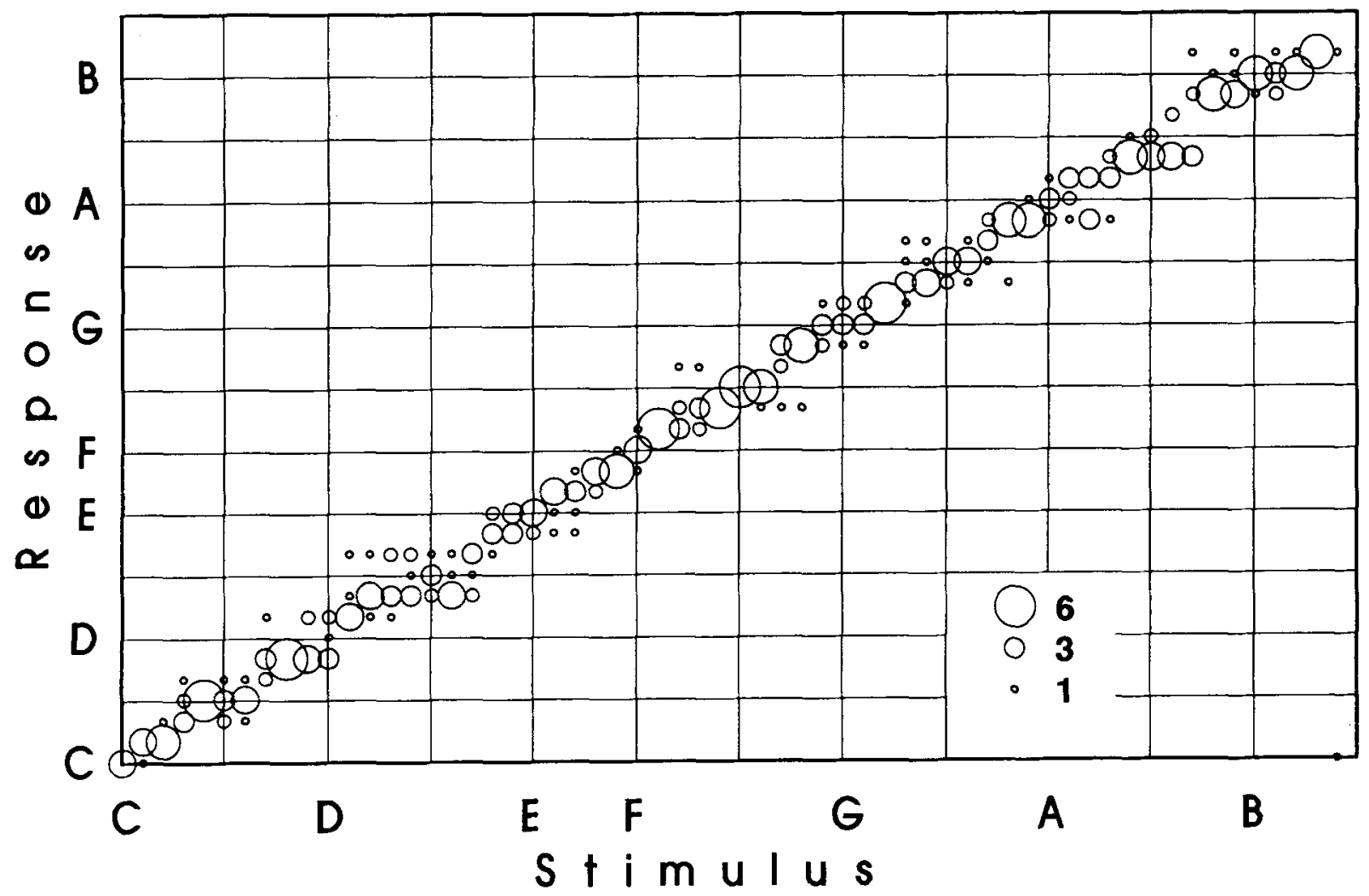

Figure 5. Scatter pattern of responses as a function of stimulus tones, viewed in the one-octave range for 1 possessor of absolute pitch (Experiment 2). The size of each circle is proportional to the number of times each response was made.

valuable information concerning the cognitive processes included in performing the task. A grand mean of the RTs was $1.629 \mathrm{sec}$ for the precise AP group (individual means ranging from 1.386 to $1.877 \mathrm{sec}), 1.966 \mathrm{sec}$ for the imprecise AP group (1.546 to $2.618 \mathrm{sec}$ ), and $2.855 \mathrm{sec}$ for the non-AP group (1.937 to $4.028 \mathrm{sec})$. An analysis of variance showed that the differences of the means among the groups were highly significant $[F(2,36)=$ $22.36, p<.0001]$. It is concluded that those who have AP can identify presented tones not only more correctly but also faster than those who have no AP.
It should be noted that time delay of the responses observed here is so long that it may be regarded as RT in the ordinary sense. This is partly because rapid responding was not the primary demand in the experiment. Instead, the subjects were requested first of all to respond as accurately as possible, and, if necessary, they were allowed to use any available cues helpful to increase response accuracy. AP possessors can easily and immediately identify the tones; however, for non-AP subjects, absolute identification is quite difficult, and to achieve a good performance, they find it necessary to rely upon

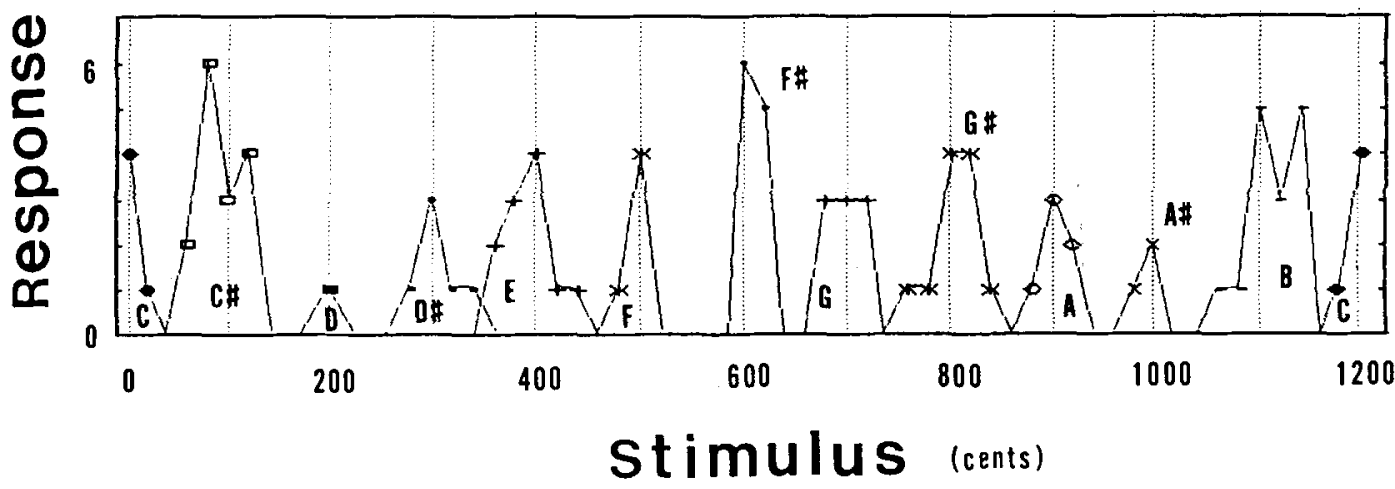

Figure 6. Number of each "in-tune" response as a function of stimulus frequencies in the one-octave range for 1 possessor of absolute pitch (Experiment 2). The stimulus frequencies are expressed in cents relative to $\mathrm{C}$ notes. 

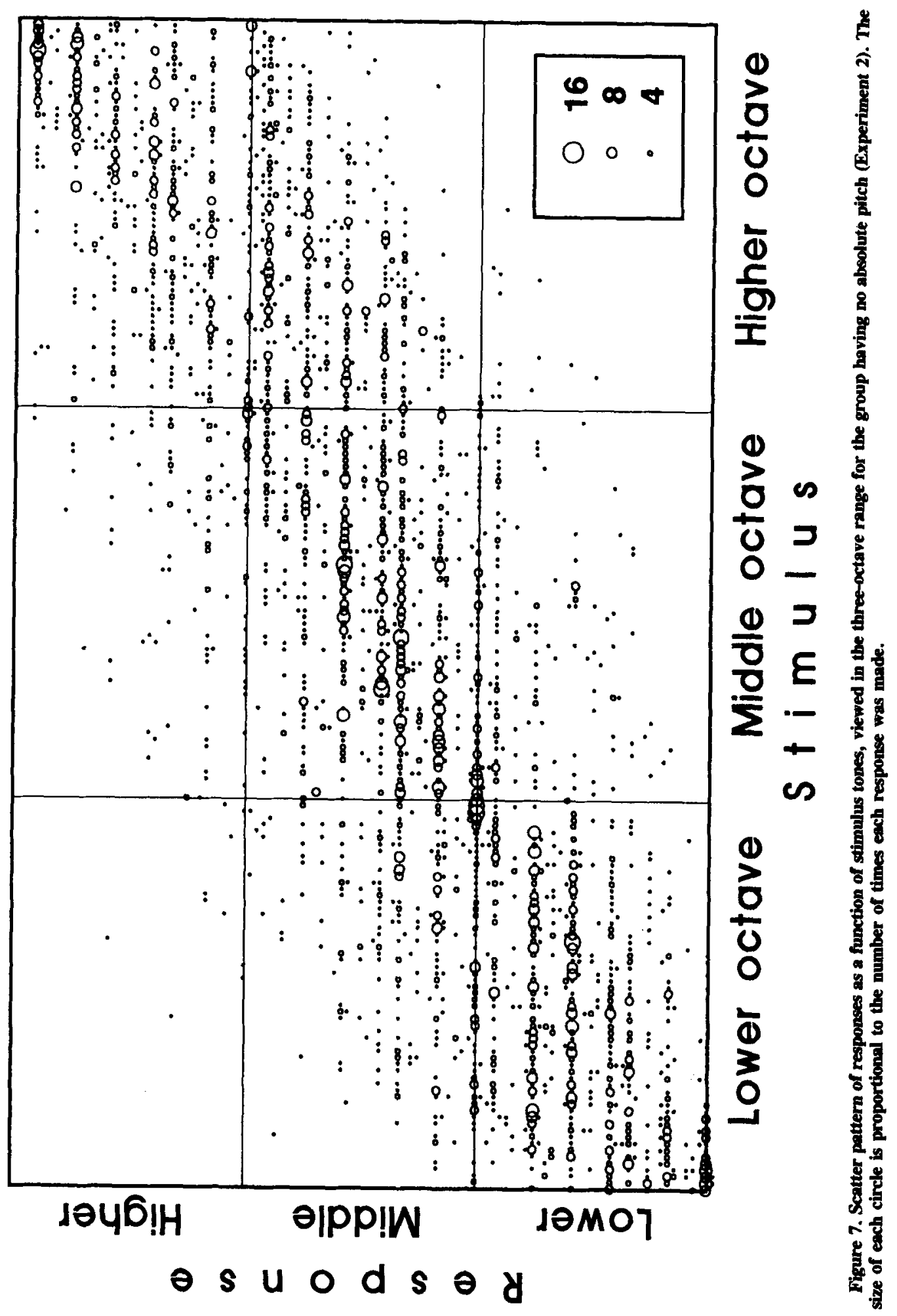
some highly sophisticated strategies (e.g., the use of relative pitch in relation to the previously presented tones or some particular pitches well memorized as references). These strategies are considered very time-consuming. Most non-AP subjects are believed to use one or more of these strategies. If fast responses are demanded first, possibly the non-AP subjects will identify the tones solely on the basis of pitch height, abandoning time-consuming strategies and resulting in faster RTs.

The mean RTs of different in-tune response categories were calculated for individuals of the precise AP group. The results of an analysis of variance showed that the effect of response categories was significant $[F(11,121)=$ $2.73, p=.0035$ ], indicating that response speeds were different for response categories. The rank order of response categories was G, C, E, D, A, F, C\#, A\#, B, $\mathrm{D} \#, F \#$, and $\mathrm{GH}$.

Interestingly enough, except for $\mathrm{B}$, the responses that designate the white-key notes on a musical keyboard (diatonic scale notes in a C-major mode) and the blackkey notes (nondiatonic scale notes in the same mode) are clearly dichotomized with respect to RT; the mean RTs of the white-key notes and the black-key notes were $1.524 \mathrm{sec}$ and $1.631 \mathrm{sec}$ for the precise AP group, and $2.733 \mathrm{sec}$ and $2.957 \mathrm{sec}$ for the non-AP group. The difference was highly significant for the precise AP group $[F(1,11)=23.17, p<.001]$, as well as for the non-AP group $[F(1,9)=6.57, p=.03]$. These results indicate that generally white-key note responses are significantly faster than black-key note responses and that this trend is stronger for the AP group than for the non-AP group. Also, the fastest responses are to tonally important notes in a C-major mode, for example, $\mathrm{G}$ (the dominant) and $\mathrm{C}$ (the tonic). If it is assumed that RT reflects accessibility to the internal representation of musical pitch, this trend in the precise AP group can be interpreted to show that AP subjects can more rapidly recognize tonally important tones in the C-major key.

There also seems to exist a consistent relationship between the RTs and the variance of responses; Pearson's correlation coefficient between them is .656. A scatterplot of the response with respect to their standard deviations and delay times is presented in Figure 8, in which the white-key notes and the black-key notes form clearly separated clusters.

On the other hand, the difference of RTs between the white-key notes and the black-key notes in the non-AP group should be interpreted differently. As noted previously, the non-AP subjects could not identify the note names at all, but categorized only roughly by using pitch height or timbre-like qualities of tones. It seems that they mainly used seven white keys, which must be enough for them as response categories. Therefore, the black-key responses, if any occurred, might be either only arbitrary ones or those made by individuals who used some of the sophisticated strategies mentioned above. In the latter case, time delays of responses using black keys are expected to be long. This inference is consistent with the

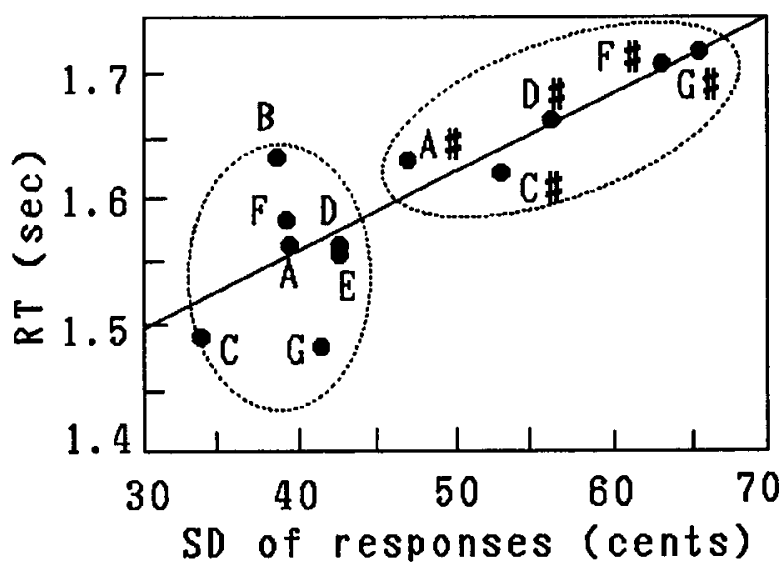

Figure 8. The relationship between standard deviations (SDs) and reaction times (RTs) of each response for the precise absolute pitch group (Experiment 2). An oblique line represents the best fitted line.

response bias that is revealed by the fact that the non-AP subjects made most responses $(85.4 \%)$ by using white keys (chance level, 58.3\%) and that 9 of 18 non-AP subjects made almost no black-key responses.

Finally, RTs of the "in-tune" responses were compared with "out-of-tune" ("higher" or "lower") ones. For the precise AP group, the mean of individual RTs was $1.571 \mathrm{sec}$ for "in-tune," $1.734 \mathrm{sec}$ for "lower," and $1.773 \mathrm{sec}$ for "higher" responses. An analysis of variance showed that differences among these responses were highly significant $[F(2,22)=14.45, p=.0001]$; tests of paired comparisons showed that the difference was significant between the "in-tune" and "higher" responses $(p<.01)$ and between the "in-tune" and "lower" ones $(p<.01)$, but the difference between the "higher" and "lower" ones was not significant. These results indicate that for AP subjects responses to in-tune categories were faster than responses to mistuned categories. A similar comparison is meaningless for the non-AP subjects, because they could not distinguish at all between in-tune and mistuned categories.

\section{GENERAL DISCUSSION}

The task of the present experiments was designed to exclude the possibility that subjects who have no AP can perform the task successfully by relying upon some trick strategies, which possibly result in outcomes apparently indistinguishable from those of genuine AP possessors. This was accomplished through several procedural devices. First, the stimuli used here were electronically generated complex tones, so that the subjects could not rely upon any timbral cues of familiar musical instruments. Second, most of the pitches deviated from standards of conventional tuning systems. These intermediate pitches between the standard scale tones were used primarily to finely determine the location and range of each musical pitch category. This also contributed toward 
making it difficult for subjects to establish any internal schema that might be used in the musical pitch judgment as a reference framework. Third, tones were dispersed over a wide pitch range, and pitch intervals between tones of two consecutive trials were at least one octave. This was to prevent subjects from relying on relative judgment from previously presented tones.

If the subjects are genuine AP possessors, these methodological devices are not necessary, because AP possessors can perceive musical quality of tones immediately without recourse to any of the tricks mentioned above. However, for non-AP subjects who have an excellent musical interval sense, these devices are necessary.

The problem of the origin of AP is still controversial. A growing body of evidence suggests that AP may be acquired effectively through learning in early life (e.g., Shuter-Dyson \& Gabriel, 1981), although in a few cases it may be inherited. The correlation between AP possession and early piano lessons reported here may be in line with this hypothesis. Surprisingly, a considerably large proportion of musically experienced subjects tested in the present experiments were AP possessors. This cannot be explained solely by inheritance, because it is known that the proportion of inherited AP is extremely low. For example, among musicians, the proportion of AP was $8.8 \%$ according to Wellek $(1963$, p. 85$)$ and $3.4 \%$ according to Révész (1913), and among nonmusicians, it was only $0.01 \%$ (Bachem, 1955). The result of an informal test of AP possession for a large number of music students performed on another occasion revealed that no less than half of them were AP possessors. Most of them had begun piano lessons as early as ages 3 to 5 . Although they had not been trained through any specially planned programs for acquiring AP, they probably acquired it from early learning. It should be noted that the acquisition of AP is dependent upon social or cultural circumstances; at least in the Japanese culture, where relatively early music lessons are to some extent widespread, an unexpectedly large proportion of music students have AP.

Next, let us consider the perceptual properties of tones in the present experiments. It has often been argued that pitch consists of two subjective properties; one is called tone height, which is represented on a rectilinear dimension from low to high, and the other is tone chroma (Bachem, 1937) or musical quality (Révész, 1913), which is represented on a circle (a chroma circle) that circulates every octave. Tone height is entirely dependent upon stimulus frequency, and for complex tones, it is characterized as a sort of timbre (a quality dependent upon frequency composition of the tones) rather than pitch. On the other hand, tone chroma has no explicit physical correlates and is considered to be a property specific to music. This idea originally was formalized by Révész (1913) as the two-component theory of pitch.

However, it can be argued that the concept of tone chroma is somewhat problematic. It often has been defined as a tonal property that corresponds to the musical note name, such as a C-like or D-like character (Révész,
1913; Wellek, 1938). According to this definition, it follows that tone chroma can be perceived only by AP possessors. In this usage of the term, tone chroma is confined to a limited case of AP and cannot be regarded as a general tonal attribute; thus, the two-component theory of pitch loses its generality. The problem seems to lie in the fact that tone chroma has tended to be defined as a special attribute perceived exclusively by AP possessors, although the two-component theory of pitch states the existence of the two components as general psychological attributes.

It is clear that many musicians without AP can identify musical characters of tones not absolutely, but relatively in a musical context. This relativity of the musical pitch (relative pitch) is much more important than AP in music. The concept of tone chroma defined in association with AP, therefore, is considered inappropriate as a general psychological attribute. Rather, it should be defined in association with relative pitch. Defined in this way, tone chroma is considered equivalent to tonality, the musical property specific to each tone in a musical context. According to this definition, one can legitimately say that a great majority of musical subjects who have no AP can perceive tone chroma in relation to other tones in a tonal context.

Along the same line of reasoning, AP possessors are peculiar in that they can perceive tone chroma absolutely in the absence of any musical context, although it is intrinsically context dependent. Due to this ability, they could produce quite consistent responses in the present experiments. On the other hand, non-AP subjects could not perceive tone chroma at all in the absence of a musical context. This is reflected in the results, which show that their responses were almost randomly dispersed within a one-octave range (Figures $3 \mathrm{a}$ and 7 ). However, because they could use other properties, such as tone height or timbral characteristics, their responses were roughly assembled around a diagonal line in a three-octave plot (Figure 7). Their performance was quite inconsistent because, in the absence of mnemonic anchors, they could use only a context-coding strategy (Durlach \& Braida, 1969).

It is interesting that response categories were differentiated with respect to accuracy and latency of response. Generally speaking, white-key note responses were more accurate than black-key note responses. This has been reported previously by several investigators (Bachem, 1950; Wellek, 1963). The present experiments showed that for the AP subjects a response to white-key notes was not only more accurate but was also faster than that to black-key notes (see Figure 8). Furthermore, responses to the important notes in a $\mathrm{C}$-major mode, for example, the tonic $\mathbf{C}$ and the dominant $\mathrm{G}$, were faster and more accurate (though less explicit). These results suggest that AP possessors cannot recognize all 12 notes equally well. Instead, they seem to recognize best the white-key notes, or perhaps only 4 or 5 important notes, from which other notes can be inferred. This brings to mind the tonal hier- 
archy observed by some investigators (e.g., Krumhansl, 1979; Shepard, 1982). However, considering that tonal hierarchy in nature refers to tonal meaning of each tone in a musical context and is, therefore, concerned with relative pitch, these results were unexpected. The dominance of the key of $C$ in the results of AP subjects may be related to the way in which they acquired AP at an early age. In the early lessons of piano playing and ear training, they usually began with playing the $\mathrm{C}$-major scale and listening to primary triads in $\mathrm{C}$ major.

It has been held that the uniqueness of AP possessors is that they have a remarkable ability to identify pitch in the absence of any contexts; however, it can be argued that they are actually unique in that they have memorized from early childhood the musical qualities of individual tones (tone chroma) in only one fixed tonal context and, therefore, have had no need to develop relative pitch sense. This may give them not only advantages, but also serious disadvantages; for example, they may have difficulty when they hear a transposed melody or when they are required to transpose a tune and sing it, because the tonal context is fixed and not transposable for them. It is quite an interesting question as to how AP possessors deal with a relative pitch task in which they are required to identify musical intervals variously transposed. Another series of experiments along this line is now in progress.

\section{REFERENCES}

ВАснем, А. (1937). Various types of absolute pitch. Journal of the Acoustical Society of America, 9, 146-151.

BАCHEM, A. (1950). Tone height and tone chroma as two different pitch qualities. Acta Psychologica, 7, 80-88.

BACHEM, A. (1955). Absolute pitch. Journal of the Acoustical Society of America, 27, 1180-1185.

BrADY, P. T. (1970). Fixed-scale mechanism of absolute pitch. Journal of the Acoustical Society of America, 48, 883-887.

BURNS, E. M., \& WARD, W. D. (1978). Categorical perceptionphenomenon or epiphenomenon: Evidence from experiments in the perception of melodic musical intervals. Journal of the Acoustical Society of America, 63, 456-468.

Chowning, J. (1973). The synthesis of complex audio spectra by means of frequency modulation. Journal of the Audio Engineering Society, 21, 526-534.

CudDY, L. L. (1968). Practice effects in the absolute judgment of pitch. Journal of the Acoustical Society of America, 43, 1069-1076.

Cuddy, L. L. (1970). Training the absolute identification of pitch. Perception \& Psychophysics, 8, 265-269.

Durlach, N. I., \& BraIDA, L. D. (1969). Intensity perception: I. Preliminary theory of intensity resolution. Joumal of the Acoustical Society of America, 46, 372-383.

KrumhansL, C. L. (1979). The psychological representation of musical pitch in a tonal context. Cognitive Psychology, 11, 346-374.

MEYER, M. (1899). Is the memory of absolute pitch capable of development by training? Psychological Review, 6, 514-516.

Miller, G. A. (1956). The magical number seven, plus or minus two: Some limits on our capacity for processing information. Psychological Review, 63, 81-96.

NEU, D. M. (1947). A critical review of the literature on "absolute pitch." Psychological Bulletin, 44, 249-266.

Petran, L. A. (1932). An experimental study of pitch recognition. Psychological Monographs, 42(Whole No. 193).

Pollack, I. (1952). The information in elementary auditory displays. Joumal of the Acoustical Society of America, 24, 745-749.

RÉvész, G. (1913). Zur Grundlegung der Tonpsychologie. Leipzig, East Germany: Veit.

SHEPARD, R. N. (1982). Structural representations of musical pitch. In D. Deutsch (Ed.), The psychology of music (pp. 343-390). New York: Academic Press.

ShUTER-DYSON, R., \& GABRIEL, C. (1981). The psychology of musical ability (2nd ed.). London: Methuen.

SiEgEL, J. A. (1974). Sensory and verbal coding strategies in subjects with absolute pitch. Journal of Experimental Psychology, 103, 37-44.

Siegel, J. A., \& Siegel, W. (1977). Absolute identification of notes and intervals by musicians. Perception \& Psychophysics, 21, 143-152. STUMPF, C. (1883). Tonpsychologie I. Leipzig, East Germany: Hirzel. WARD, W. D. (1963). Absolute pitch: Part II. Sound, 2, 33-41.

WARD, W. D., \& BurNs, E. M. (1982). Absolute pitch. In D. Deutsch (Ed.), The psychology of music (pp. 431-451). New York: Academic Press.

WeDELL, C. H. (1934). Nature of absolute judgment of pitch. Journal of Experimental Psychology, 17, 485-503.

WelleK, A. (1938). Das absolute Gehör und seine Typen. Zeitschrift für angewandte Psychologie, 83, 1-368(Suppl.).

Wellek, A. (1963). Musikpsychologie und Musikdsthetik: Grundriss der systematischen Musikwissenschaft. Frankfurt, West Germany: Akademischer Verlag.

(Manuscript received October 15, 1987; revision accepted for publication June 16,1988 .) 